\title{
Performance of the angle of arrival correction system of the I2T + ASSI stellar interferometer
}

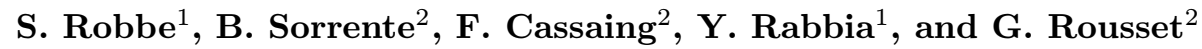 \\ 1 Observatoire de la Côte d'Azur, Fresnel Department, UMR 6528, Av. Copernic, 06130 Grasse, France \\ 2 ONERA, 29 Av. de la Div. Leclerc, BP. 72, 92322 Châtillon Cedex, France
}

Received October 9; accepted December 23, 1996

\begin{abstract}
The Active Stabilization in Stellar Interferometry (ASSI) recombining table, developed at Office National d'Etudes et de Recherches Aérospatiales (ONERA), France, was implemented on the two-Telescope Interferometer (I2T) of the Observatoire de la Côte d'Azur (OCA) in 1993, compensating for the atmospheric turbulence wavefront fluctuations. Initial fringe observations were made in June 1994 with a 11 m North-South baseline. In this paper, we report the performance of the ASSI table servo system for correction of the angle of arrival. Tracking accuracy of about \pm 0.24 arcsec was achieved in typical seeing conditions $\left(r_{0} \geq 10 \mathrm{~cm}\right)$ and on $m_{v}=2$ sources. Collected data allows to compare theoretical with observed temporal power spectra of the angle of arrival fluctuations, using Kolmogorov model. Spatio-temporal seeing parameters met during observations (Fried parameter $r_{0}$ and coherence time $\left.t_{0}\right)$ are estimated.
\end{abstract}

Key words: atmospheric effects - instrumentation: interferometers - methods: observational — methods: data analysis - techniques: interferometric

\section{Introduction}

The two-telescope interferometer (I2T) (Koechlin 1985) located at Observatoire de la Côte d'Azur (OCA), France, works with two 26-cm telescopes movable along a NorthSouth baseline. The I2T built by Antoine Labeyrie in 1972 for observing interference fringes on $\alpha$ Lyrae with a 12 $\mathrm{m}$ baseline (Labeyrie 1975), provided high angular resolution measurements in the visible and near infrared domains (Di Benedetto \& Rabbia 1987; Koechlin \& Rabbia 1985; Faucherre et al. 1983; Bonneau 1979). In 1993, a new beam combining table, the Active Stabilization in Stellar Interferometry (ASSI) table (Damé et al. 1988; Sorrente et al. 1991), developed at Office National d'Etudes et de

Send offprint requests to: S. Robbe (robbe@ocar01.obs-azur.fr)
Recherches Aérospatiales (ONERA), France, was installed at I2T (Robbe et al. 1994). The goal of this instrument is to improve the quality of interferometric measurements in routine operation of the interferometer.

ASSI is a recombining table for automatic acquisition of the interference fringes and compensation for the optical path fluctuations, a task for which it is crucial to minimize the wavefront distortions caused by atmospheric turbulence. Most importantly, the mean slope of the wavefront over each aperture must be stabilized and the remaining corrugations have to be minimized. Such stabilizing is achieved by correcting the angle of arrival. To keep the loss of fringe visibility below $5 \%$, the required single beam stability must correspond to residual fluctuations of less than 0.12 of the size of the Airy disk radius (Buscher 1988). The first fringes were observed and stabilized on the ASSI table in June 1994 with a $11 \mathrm{~m}$ baseline.

The instrument is described in Sect. (2), providing specific requirements linked to the operating conditions for the system so as to reach the expected tracking accuracy. In Sect. (3), we recall what is the available information from observed data, namely the characteristics of the servo-system, the estimation of the Fried parameter $r_{0}$ and of the temporal spectrum of the angle of arrival fluctuations. The measurements are discussed in Sect. (4), and Sect. (5) provides results concerning observed tracking accuracy, estimation and statistics of seeing parameters, and observed temporal power spectra of the angle of arrival fluctuations. The latter is compared with the behavior predicted by Kolmogorov theory.

\section{Description of the instrument}

\subsection{The I2T interferometer}

Figure 1 shows a schematic view of I2T (Koechlin \& Rabbia 1985). It consists of two $26 \mathrm{~cm}$ diameter telescopes mounted on rail tracks. Output parallel beams are directed toward a central building (horizontal propagation) where they are combined (Schumacher et al. 1991). To 
compensate for the optical path difference (OPD) due to Earth rotation, a delay line is inserted in the Southern arm of the interferometer, by means of a cat's eye system. This delay line is movable under computer control with a $1 \mathrm{~m}$ translation stroke (corresponding to $2 \mathrm{~m} \mathrm{OPD}$ ) with a $1 \mu \mathrm{m}$ resolution (Koehler \& Manil 1990). The Northern arm is equipped with a fixed delay line that merely ensures optical and metrological symmetry. The movable Southern delay line was built in collaboration with AérospatialeCannes and the Northern cat's eye in collaboration with Matra.

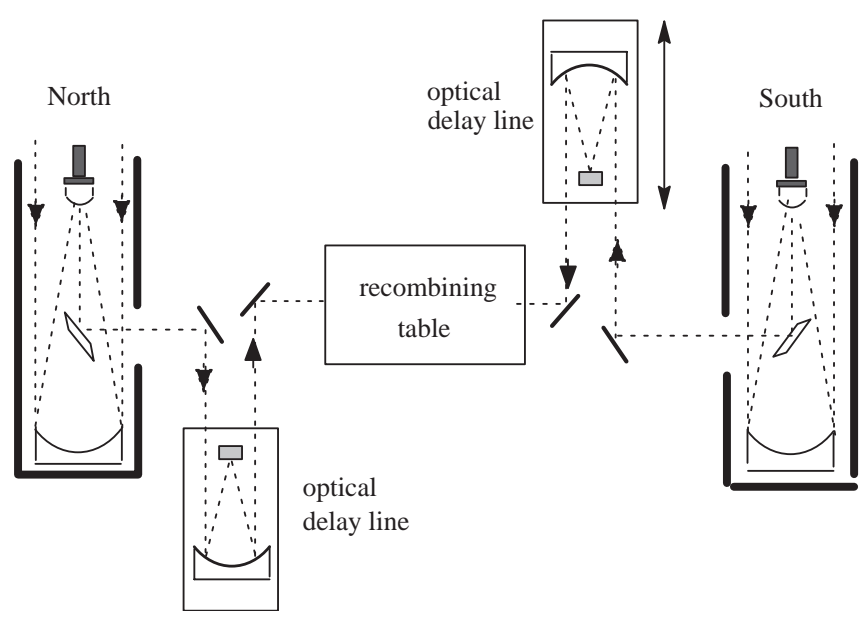

Fig. 1. Schematic view of the I2T interferometer

As soon as the diurnal OPD is removed, residual OPD fluctuations can be corrected. They are mostly induced by atmospheric turbulence and also by mechanical instabilities along the light paths. Note that due to the seeing conditions on this site, the telescopes are practically never diffraction-limited at visible and near infrared wavelengths $(\lambda<1 \mu \mathrm{m})$.

\subsection{The ASSI table}

The main constituents of ASSI are (see Fig. 2):

- The star tracker, including two active tip-tilt mirrors.

- The fringe tracker, including an OPD fluctuation compensation capability.

- The dispersed fringe analysis channel, used as the science instrument.

The star tracker compensates for fluctuations of the wavefront angle of arrival, and is designed to keep the two interfering wavefronts parallel to within a fraction of one arcsecond. It is described in Sect. 2.3.

The fringe sensor allows automatic fringe detection and provides an error signal used for fringe stabilization.

Since the wavefront distortions are larger in the visible than in the infrared, the fringe tracking system has

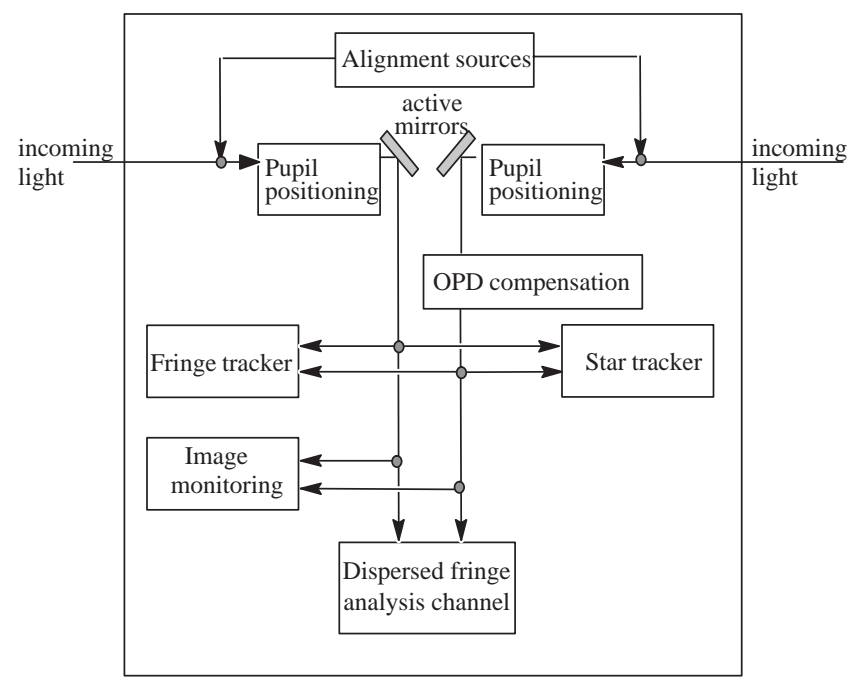

Fig. 2. Functional architecture of the ASSI table

been chosen to operate in the near infrared $(0.78 \mu \mathrm{m}$ to $1.05 \mu \mathrm{m})$. Both pupils are superimposed on a photon counting avalanche photodiode (APD). The other flat-tint output of the Mach-Zehnder interferometer feeds an intensified CCD camera for pupil control. The three unknowns of the interferogram (intensity, visibility and phase) are measured with a temporal sine modulation and a synchronous demodulation by a specific real time algorithm (Cassaing et al. 1995). The setup is roughly similar to the Mark III interferometer (Shao et al. 1988). Only one broadband spectral channel is used, the coherence length of which is tuned by a spectral filter. Calculated visibilities are used in automatic fringe detection during a computercontrolled OPD scan. This automatic acquisition capability is a marked improvement over visual fringe acquisition as on the previous I2T (Koechlin \& Rabbia 1985).

OPD correction is performed from the calculated phase by a two-stage fine delay line. A piezoelectric transducer (PZT) with $\pm 16 \mu \mathrm{m}$ OPD dynamic range allows high frequency correction and high OPD resolution, while a motorized table with $\pm 2 \mathrm{~cm}$ OPD range is used for PZT desaturation when it exceeds a given position. The main drawback of this system is that the calculated phase suffers from classical $2 \pi$ wrapping (phase tracking mode), whereas other interferometers are now relying on another estimator with a wider dynamic (envelope tracking mode). OPD correction is therefore limited by fringe jumps.

The dispersed fringe analysis channel allows to record fringes in image plane $(384 \times 288$-pixel intensified CCD camera) at a rate of 50 frames per second.

The basic feature is the dispersion of the fringe pattern leading to successive adjacent spectral channels each roughly $1.1 \mathrm{~nm}$ in width. Thus, a large bandwidth is available without reducing the coherence length (about $300 \mu \mathrm{m}$ ) since the latter is determined by the width of an individual channel. Up till now, the major limitation to 
accuracy of visibility measurements in this channel has been atmospheric degradation. The star tracker is expected to improve the superimposition of the image patches on the CCD camera so that fringes do not disappear because of poor overlapping, and the fringe tracker is expected to remove the fringe random motion to improve the accuracy of the measured fringe visibilities. Longer integration time can then be expected, so that magnitudes larger than the ones observed in the past could be reached. Three auxiliary modules are also available: 1 ) on a monitoring camera, the two stellar images are observed in a \pm 15 arcsecond field, enabling quick detection of such problems as optical aberrations (telescope defocus for example), photometric dissymmetry or bad seeing conditions ; 2) auxiliary sources allow internal alignment of the table and external alignment of the pupils prior to each observation, and are used to determine the internal zero OPD of the table (Robbe et al. 1994); 3) the pupil positioning subsystem allows pupil positions to be kept constant inside the table. Optimal working conditions dictate that pupil image be kept accurately centered on the active mirror irrespective of telescope position on each arm. This is achieved by a set of zoom-forming optics movable on each arm as a function of baseline.

\subsection{The star tracker}

\subsubsection{Description of the system}

The angle of arrival correction system is made of several parts (see Fig. 3).

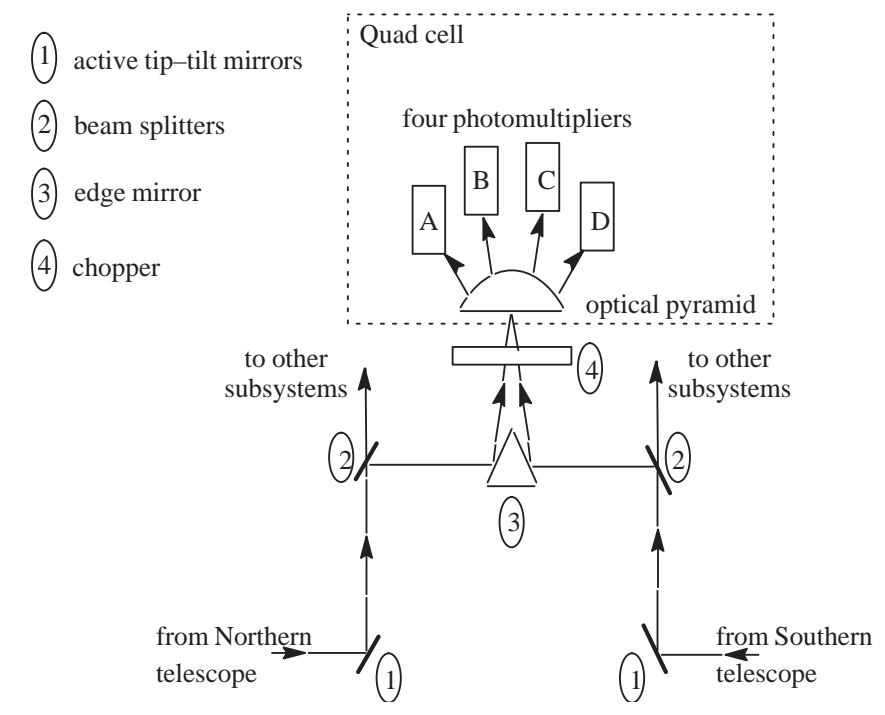

Fig. 3. Star tracker

a) The quad cell

Half of the visible light $(0.4 \mu \mathrm{m}$ to $0.78 \mu \mathrm{m})$ is beam-split from each stellar beam (North and South) and sent toward a reflecting pyramid in a focal plane where the stellar spot is divided into four parts. Each part of the spot is directed to a GaAs photon-counting photomultiplier (PMT). The quad cell is common to both beams: a temporal multiplexing is performed by a rotating chopper which allows to alternately reimage on the quad cell the observed star from the two telescopes. The integration time is $3 \mathrm{~ms}$ successively for each beam, as against a sampling interval of $6 \mathrm{~ms}$. The drawback of the reduced throughput is that the quad cell is cheaper and that differential angle of arrival cannot arise from drifts in the quad cell.

b) The calculator

Pulses from the four PMTs which exceed a threshold are synchronously counted with the chopper. At the end of each integration time, an analog calculator computes the angular error along each axis from the content of the four counters $A, B, C$ and $D$ converted to analog signals. The two errors $\varepsilon_{x}$ and $\varepsilon_{y}$ are defined by:

$$
\begin{aligned}
\varepsilon_{x} & =(A+B-C-D) /(A+B+C+D) \\
\text { and } \varepsilon_{y} & =(A+C-B-D) /(A+B+C+D) .
\end{aligned}
$$

This calculator sends offsets to the telescopes when the active mirrors reach user-defined bounds (correction of telescope drifts).

c) The mirrors

The two tip-tilt voice-coil mirrors were made by MicroControle. They are servo-controlled by an internal position sensor and their characteristics are as follows:

- clear aperture: $50 \mathrm{~mm}$

- stroke: $\pm 6.4 \mathrm{mrad}$

- resolution: $1 \mu \mathrm{rad}$

- bandwidth: $250 \mathrm{~Hz}$ at $-3 \mathrm{~dB}$.

This high closed loop bandwidth hides the fact that a mechanical resonance appears at $250 \mathrm{~Hz}$ and that the phase of the transfer function exhibits a quick linear drift $\left(30^{\circ}\right.$ at $50 \mathrm{~Hz})$.

Taking into account the angular magnification of the ASSI table, the stroke reported on the sky is \pm 12 arcsec and the resolution is 2 arcsec. The small size of the pupil on the active mirrors $(1.4 \mathrm{~mm})$ is not well suited. It was chosen for previous less efficient mirrors and by the constraint of reimaging the distant telescope pupil on the mirror.

For star tracking, the error signal from the internal position sensor is replaced by the error measured by the quad cell. An analog tunable gain allows the user to optimize the bandwidth for each axis, according to turbulence and noise conditions.

d) The interface

The calculator and the mirrors are interfaced with a PC in charge of the whole ASSI operation (user interface and fringe tracking computation). This PC allows to initialize the analog calculator, handshakes with the telescopes for 
star acquisition, moves the mirrors for optical adjustment or on-line calibration, controls the chopper, stores files of data with either the 8 PMT counts or the 4 quad cell signals or the 4 mirror positions.

\subsubsection{Characteristics of the servo system}

In closed loop, the residual error is limited by the quad cell noise, the mirror transfer function phase rotation and the quad cell sampling rate. A bandwidth of $63 \mathrm{~Hz}$ in closed loop at $-3 \mathrm{~dB}$ can be reached at high light level with the $330 \mathrm{~Hz}$ chopper (165 Hz sampling frequency). Figures 4 to 6 show the open loop transfer function, the closed loop transfer function and the rejection transfer function, determined by numerical simulations (based on the temporal characteristics of the loop including a $1.5 \mathrm{~ms}$ mean delay and the mechanical modeling of the mirror) and consistent with the laboratory performance. The closed loop transfer function shows the ratio between the mirror position and the atmospheric perturbation (Demerlé et al. 1994). In the low frequency domain, the mirror follows (corrects) the perturbation. In the high frequency domain, beyond the cutoff frequency, the correction is inefficient. Between the two, there is a resonant regime in which the mirror movement is slightly stronger than the perturbation.

The closed loop transfer function (in modulus) does not show the residual error between the perturbation and the correction, which creates a residual error. The relevant curve is the rejection transfer function which plots the ratio of the residual error to the incoming perturbation (Fig. $6)$. Up to the $0 \mathrm{~dB}$ bandwidth $(22 \mathrm{~Hz})$, the perturbation is attenuated: this is the goal of the servo system. Beyond the bandwidth, the perturbation is unaffected, or even amplified near the resonance. For optimum operation, since atmospheric perturbation is mainly concentrated in low frequencies, the rejection bandwidth should be greater than the atmospheric cutoff frequency (see Sect. 3).

\subsubsection{Quad cell response analysis}

As given by Eq. (1), the position of the star image is given by a combination of the four integrated intensities. Assuming small image displacement and image size much smaller than quadrant size, the position error is related to the image position $\left(x_{i}, y_{i}\right)$ by an expression of the form:

$\varepsilon_{x}=\frac{2 x_{i}}{w_{i}}$ and $\varepsilon_{y}=\frac{2 y_{i}}{w_{i}}$

where $w_{i}$ is the full width at half maximum (FWHM) of the star image. The angle of arrival $\left(a_{x}, a_{y}\right)$ can therefore be calculated by:

$a_{x}=\frac{w_{i} \varepsilon_{x}}{2 f G}$ and $a_{y}=\frac{w_{i} \varepsilon_{x}}{2 f G}$

where $f$ is the focal length of the optical system and $G$ the magnification. Let us underline that to convert position error into an angle, the star image FWHM must be
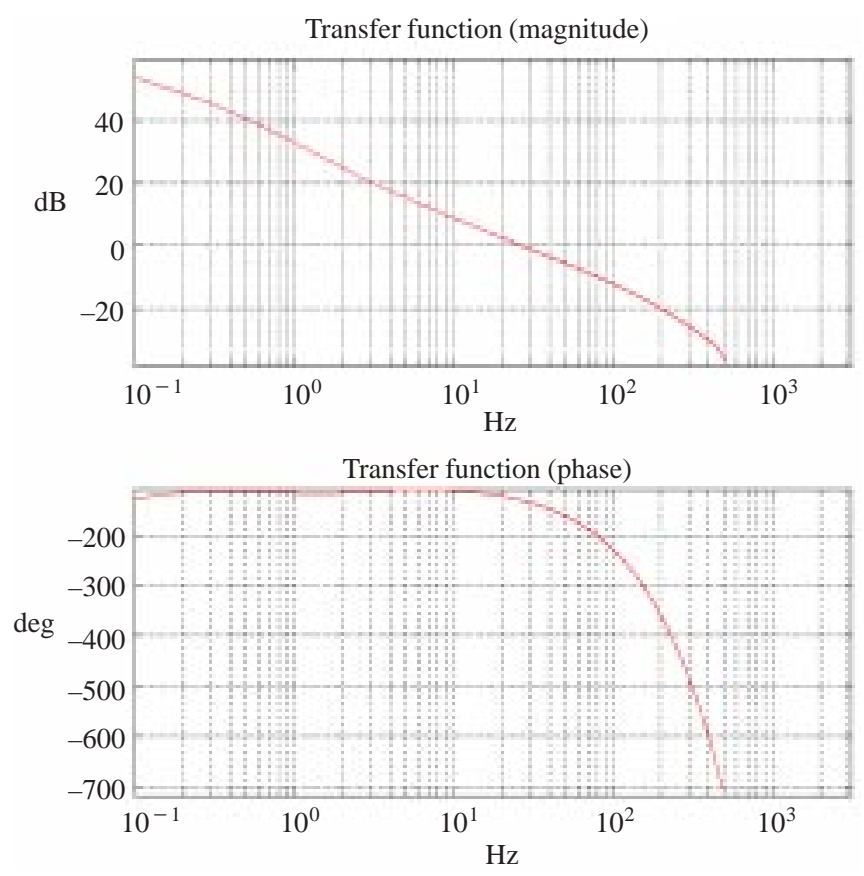

Fig. 4. Open loop transfer function determined by numerical simulations
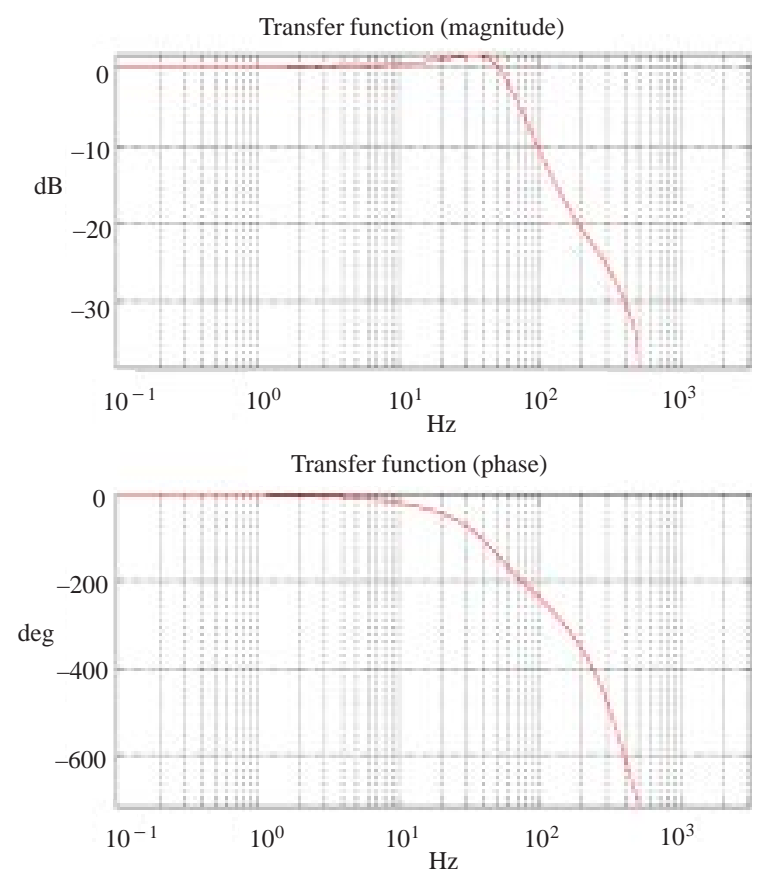

Fig. 5. Closed loop transfer function determined by numerical simulations 

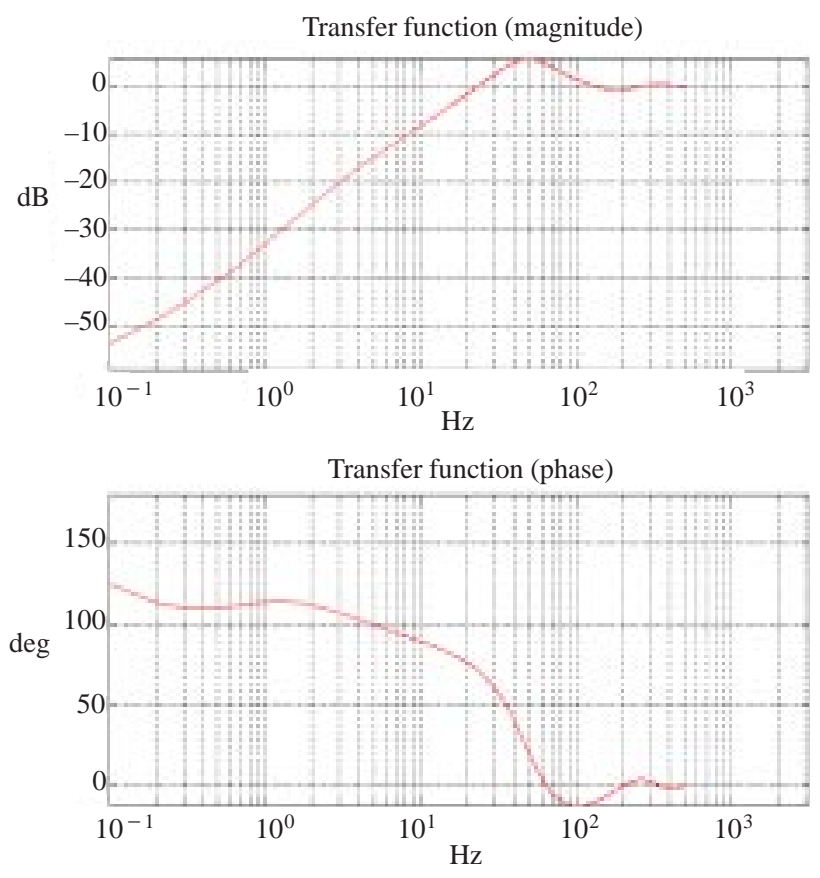

Fig. 6. Rejection transfer function determined by numerical simulations

known. This is the case, for instance, if the star image is unresolved and limited by the telescope diffraction. The image size is then given by:

$w_{i}=\frac{\lambda f G}{D}$

where $D$ is the telescope diameter.

But, with the ASSI + I2T experiment, the telescope diameter is often larger than $r_{0}$ in the spectral band of the star tracker system. The star image is therefore seeing limited. The FWHM is roughly given by (Yura 1973):

$w_{i}=\frac{\lambda f G}{D} \sqrt{1+\left(\frac{D}{r_{0}}\right)^{2}}$

$w_{i}$ depends on the seeing conditions, and the quad cell response too (Ma et al. 1989). Therefore, in such a case, $w_{i}$ is unknown.

The problem induced by the unknown FWHM is uncertainty as to the value of the loop gain of active control (Eq. (3)). Therefore the loop gain has to be calibrated on the star image itself before closing the loop, and readjusted during observation. An alternative way is to defocus the star image on the quad cell in order to keep a constant spot size.

Yet another problem affects the quad cell response: the position measurement is nonlinear for a wide angle. Equation (2) is only valid for small displacements. For a wide angle, the signal saturates (Gerson \& Rue 1989).

As we just mentioned, optimization of the control loop must take into account the specific conditions of turbulence met during a given observation, and this of course cannot be achieved once for all at the internal optimization stage. To that aim, an additional (analog) gain has been included in the control loop, and has to be time-adjusted in order to preserve the optimal characteristics. Criterion of this adjustment is the minimizing of the observed standard deviation of the residual error $\varepsilon(t)$, recorded during a few tens of seconds. It turned out that the response of the control loop is pretty sensitive to this adjustment which should be made frequently in the course of observation in connection with the time evolution of the turbulence parameters, Fried parameter $r_{0}$ and coherence time $t_{0}$. As an example, Fig. 7 shows two sequences of residual errors on the $X$ and $Y$ axes of the Northern mirror obtained over 10 seconds on the 13th of December 1994, respectively in open loop and in closed loop mode, with adjusted servocontrol gains. In open loop, the spot diagram size is about 0.9 arcsec while, in closed loop, it is about 0.3 arcsec. These values show the importance of the servo tracker in terms of minimizing the angle of arrival effect.

\subsubsection{Quad cell measurement noise}

We present here the expression of the measurement error due to different sources of noise like photon noise, background and electronic noise. Let $N_{\mathrm{ph}}$ be the number of detected photons per telescope during the exposure time, we have $N_{\mathrm{ph}}=A+B+C+D$. The expressions of the angle of arrival variance are deduced from Eqs. (1), (3) and (5). For photon noise, we have:

$\operatorname{Var}(\alpha)=\frac{1}{4}\left(\frac{\lambda}{D}\right)^{2}\left(1+\left(\frac{D}{r_{0}}\right)^{2}\right) \frac{1}{N_{\mathrm{ph}}}$

and for electronic noise:

$\operatorname{Var}(\alpha)=\left(\frac{\lambda}{D}\right)^{2}\left(1+\left(\frac{D}{r_{0}}\right)^{2}\right) \frac{\sigma_{\mathrm{e}}^{2}}{N_{\mathrm{ph}}^{2}}$

where $\sigma_{\mathrm{e}}$ is the number of electrons of noise per quadrant. For background noise, Eq. (7) can be used with $\sigma_{\mathrm{e}}^{2}$ as the number of background detected photons. Equations (6) and (7) are similar to the one given by Tyler \& Fried (1982). They are also relatively close to the expressions derived in the case of a Shack-Hartmann wavefront sensor (Rousset 1994).

We observe that the spread of the star image due to the seeing severely limits the sensitivity of the quad cell detector. The best performance is obtained for diffractionlimited images. For the I2T + ASSI experiment generally $D>r_{0}$. Thus, measurement noise depends on seeing conditions. 

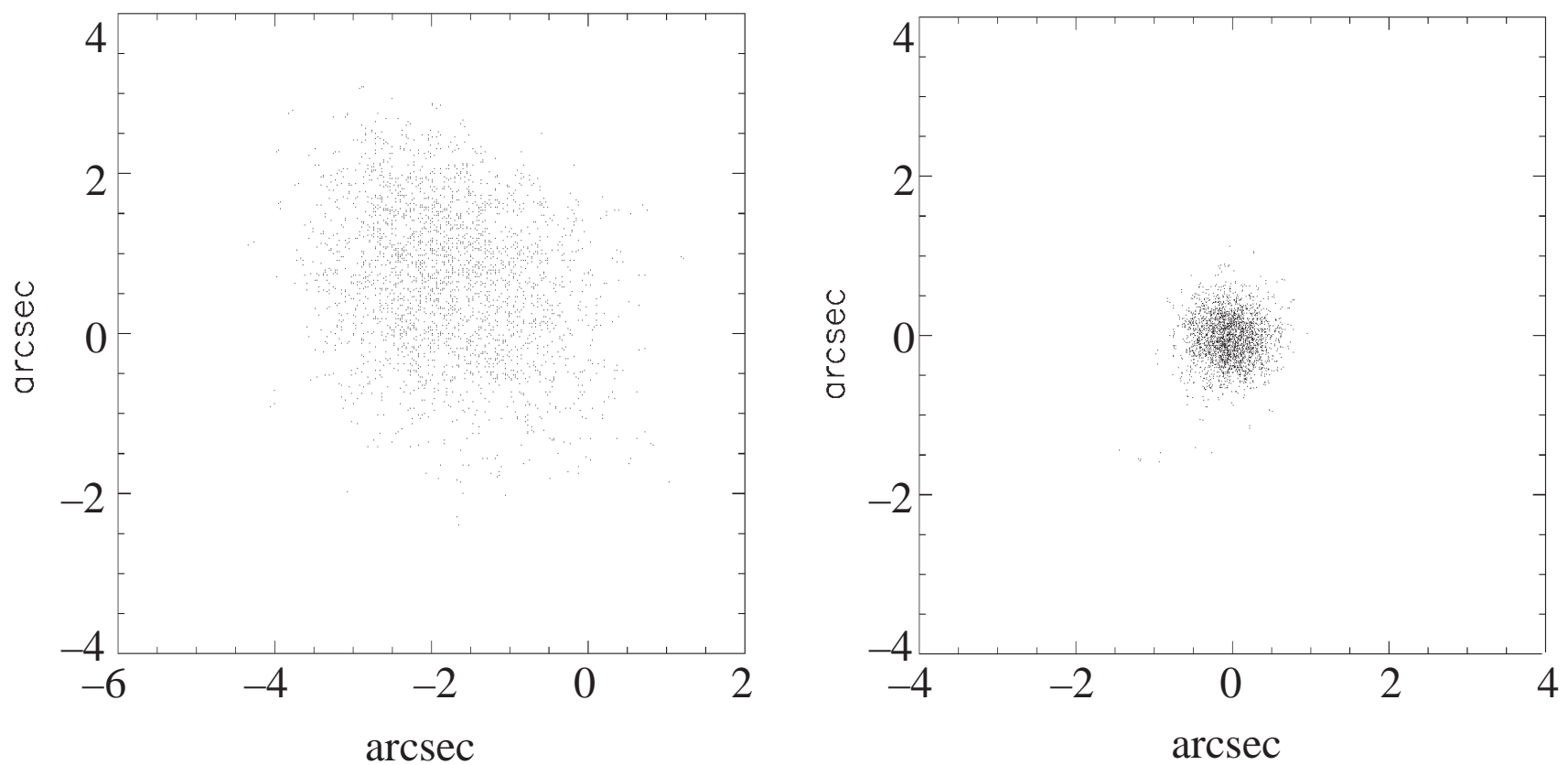

Fig. 7. Open loop and closed loop residual errors on the $X$ and $Y$ axes of the Northern mirror with adjusted servo-control gains $(12 / 13 / 94-10$ seconds)

\section{Angle of arrival characteristics}

\subsection{Temporal characteristics}

\subsubsection{Temporal power spectrum}

The angle of arrival is defined as being the normal direction relative to the wavefront mean surface, at the point of observation and can be expressed as the mean value of the derivative of the disturbed wavefront at a given aperture (Roddier 1981). The angle $\alpha$, measured at any given point on the ground, is therefore the convolution of the pupil function with this derivative:

$\alpha(\bar{r})=\Pi\left(\frac{\bar{r}}{D}\right)^{*} \frac{\delta \phi(\bar{r})}{\delta x}$

where $\alpha$ is the component of the angle of arrival on the $x$ direction, $\phi$ the phase of the disturbed wavefront, and $\Pi$ the pupil function equal to zero outside the aperture. $\alpha$ is a random variable that can be characterized by its temporal power spectrum $w_{\alpha}(\nu)$, where $\nu$ is the temporal frequency. To derive the expression of $w_{\alpha}(\nu)$, the outer scale $\mathcal{L}_{0}$ is taken as infinite for convenience. Its size remains a subject of controversy, estimations ended up to values from a few meters (Coulman et al. 1988) to several kilometers (Colavita et al. 1987). Moreover, it is believed to largely depend on meteorological conditions. The temporal power spectrum $w_{\alpha}(\nu)$ for a one-layer atmosphere can then be written under Taylor hypothesis as follows (Conan 1994):

$$
\begin{aligned}
& w_{\alpha}(v)= 0.078(2 \pi)^{4 / 3} \frac{v^{2}}{\mathcal{V}^{3}} r_{0}^{-5 / 3} \int_{-\infty}^{+\infty}\left|\frac{2 J_{1}(\pi D f)}{\pi D f}\right|^{2} \\
& f^{-11 / 3} \mathrm{~d} f_{y}
\end{aligned}
$$

$\mathcal{V}$ is the average wind speed on the $x$-axis, $J_{1}$ is the Bessel function of order 1 , and $f$ is the module of the spatial frequency $\vec{f}, f=\sqrt{\frac{v^{2}}{\mathcal{V}^{2}}+f_{y}^{2}}$.

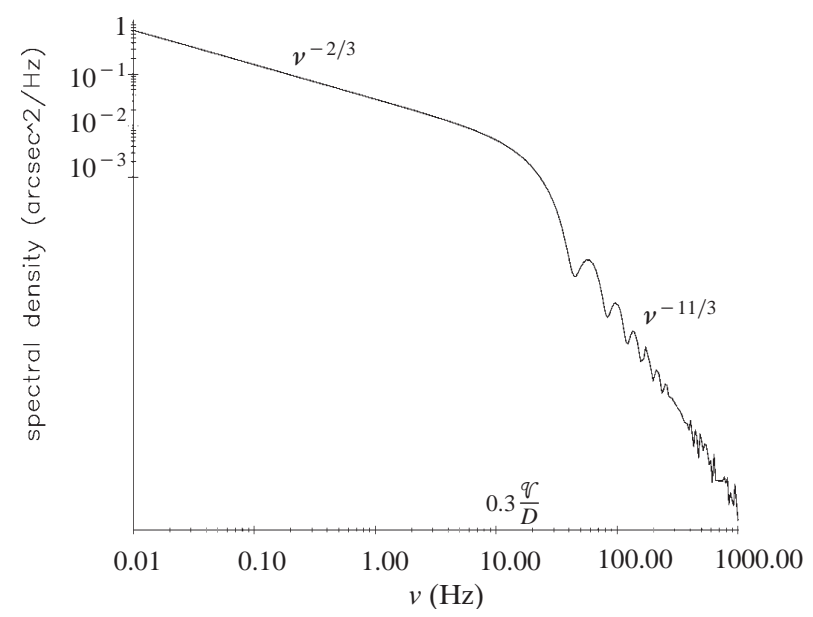

Fig. 8. Normalized theoretical power spectrum, $0.3 \mathcal{V} / D=11.5 \mathrm{~Hz}$

Figure 8 shows the spectrum obtained by numerical integration of Eq. (9) with the following values ascribed to the 
parameters: aperture diameter $D$ measures $26 \mathrm{~cm}$, Fried parameter $r_{0} 10 \mathrm{~cm}$, and wind velocity $\mathcal{V}$ on the $x$-axis $10 \mathrm{~m} / \mathrm{s}$. The temporal power spectrum of the angle of arrival shows a $\nu^{-2 / 3}$ power law in the low frequency domain, while in the high frequency domain, the spectrum decreases as $\nu^{-11 / 3}$ (Hogge \& Butts 1976; Martin 1987; Conan et al. 1995). A power law in $\nu^{-17 / 3}$ is mentioned for the tilt by Roddier et al. (1993) and Conan (1994) and it concerns the phase component on Zernike modes z1 and z2. ASSI measures the angle of arrival, expressed as the mean value of the phase first derivative, implying the $\nu^{-11 / 3}$ power law. In Fig. 8, a modulation of the profile appears in the high frequency part of the spectrum. This is due to oscillation induced by the term containing the Bessel function $J_{1}$ (Conan 1994). Noise appearing over this modulation is likely to be induced by the numerical tool. The spectrum is characterized by a frequency (knee frequency) at which a slope-break marks the separation between the two domains. This knee frequency $\nu_{\text {knee }}$ is given by the empirical formula (Conan et al. 1995):

$\nu_{\text {knee }} \approx 0.3 \frac{\mathcal{V}}{D}$

For the chosen values of $\mathcal{V}$ and $D, \nu_{\text {knee }}$ equals $11.5 \mathrm{~Hz}$. In practice, the parameter $\mathcal{V}$ is the mean velocity within the different layers of the turbulent atmosphere met by the wavefront. Wind direction and amplitude fluctuations from a layer to another can induce change around the knee frequency but the asymptotic behaviors are not modified (Conan et al. 1995). Therefore from experimental power spectrum knee frequency, the average wind speed in the atmosphere can be estimated.

\subsubsection{Coherence time}

Estimates of the coherence time $t_{0}$ are available as a result of the Fried parameter and wind velocity estimations. Knowledge of these parameters is essential in determining temporal bandwidth of optical systems and integration times in interferometric imaging. Using wind speed and $r_{0}$ estimates obtained from angle of arrival temporal spectra, $t_{0}$ is given by the formula (Nightingale \& Buscher 1991; Davis et al. 1995; Davis \& Tango 1995):

$t_{0}=0.314 \frac{r_{0}}{\mathcal{V}}$

Equation (11) is only valid in the inertial range of Kolmogorov (Roddier 1981).

We must note that the optics community has not concurred over a single definition of the coherence time. Colavita et al. (1987) define a coherence time $T_{0}$ such that $T_{0}=0.81 \frac{r_{0}}{\mathcal{V}}$.

\subsection{Spatial characteristics}

\subsubsection{Image tracking accuracy}

Reducing the angle of arrival fluctuations results in stabilization of image position, and the residual position error $\varepsilon(t)$ expressed in arcsecond on the sky can be identified as the observable residual motion of the image. In this paper, performance of the system is traced by the standard deviation $\sigma_{\varepsilon}$ of $\varepsilon(t)$, which can be taken as a measure of the tracking accuracy. It can be directly obtained from raw data, or from the integral of the power spectrum.

\subsubsection{Fried parameter}

Since $r_{0}$ is directly related to the standard deviation $\sigma_{\alpha}$ of the image motion (angle of arrival) on one axis, it can be written as follows (Tatarski 1971):

$r_{0}=\left(0.975 \frac{3.44}{2 \pi^{2}}\right)^{\frac{3}{5}} D\left(\frac{\lambda}{\sigma_{\alpha} D}\right)^{\frac{6}{5}}$

\section{Analysis of the measurements}

\subsection{Turbulence measurements}

The first goal of the star tracker is to provide image stabilization for better measurement of astrophysical quantities. But correction of atmospheric fluctuations also yields information about turbulence. The Fried parameter $r_{0}$ can be estimated from the angle of arrival variance according to Eq. (12) In this paper, $r_{0}$ values are given at $600 \mathrm{~nm}$, the mean wavelength of the star tracker system.

The atmospheric perturbation can be measured either in open loop from the quad cell signal, or in closed loop from the mirror position. In both cases, these data include mechanical vibrations inside the instrument and telescope drift, and cannot therefore provide accurate $r_{0}$ measurement, but rather qualitative estimation of the strength of the turbulence to be corrected. Since the correction is not perfect, mirror position reflects the atmospheric angle of arrival filtered by the closed loop transfer function, whose cutoff frequency is close to the atmospheric one $(63 \mathrm{~Hz}$ in comparison with $11.5 \mathrm{~Hz}$ ), see Figs. 5 and 8. Deconvolution with the servo transfer function would therefore be interesting for a better estimate, but not implemented for the results of this paper. This closed loop mode is used for a posteriori $r_{0}$ calculation $\left(r_{0 \mathrm{CL}}\right)$, from files of mirror position in which the (assumedly linear) telescope drift was subtracted.

Since the sampling frequency of the quad cell is much higher $\left(165 \mathrm{~Hz}\right.$ in open loop), a real-time $r_{0}$ estimation $\left(r_{0 \mathrm{OL}}\right)$ can be made from the quad cell data in open loop mode. The drawback in this case is that the linear area of the quad cell roughly equals the size of the focal spot: the star must be centered and only small angles of arrival can be directly measured. Nevertheless, this mode has been 
implemented on ASSI, with a very low frequency correction to keep the spot centered. We assume that the correction is performed in the linear area of the quad-cell. The quad-cell calibration is automatically performed before data acquisition and is fulfilled again during the observations, when the turbulence conditions change and when the image size varies on the ecartometer as a function of $\lambda / r_{0}$ (details in Sect. 2.3.3). This correction compensates for the telescope drift during the long time scale measurement, but low atmospheric frequencies are corrupted.

\subsection{Conditions of data recording}

Data used to evaluate the power spectra of the angle of arrival and of the image tracking accuracy were acquired during a time period of several tens of seconds at a frequency rate higher than the sampling frequency of the servo loop. Typically, the number of data per channel is equal to 8400 in the case of a 30-second recording. The fluctuations of frequency below about $0.03 \mathrm{~Hz}$ are therefore filtered by the record process.

The command period of the servo system is equal to $T_{\mathrm{e}}=6 \mathrm{~ms}$. So, according to Shannon conditions, the angle of arrival cannot be determined at upper frequencies than $1 / 2 T_{\mathrm{e}}=82.5 \mathrm{~Hz}$.

\subsection{Theoretical accuracy}

According to the theoretical transfer functions of the servo system (Figs. 4 to 6 ) simulations of the performance of the servo-system have been done. Photon flux close to the values measured during the observations has been taken (see the following section). A theoretical power spectrum of the angle of arrival characterized by a $-2 / 3$ slope in the low frequency domain and a $-11 / 3$ slope in the high frequency domain has been used to represent the incoming perturbation in the servo system. The results of the simulations are presented in Fig. 9, which shows the variation of the relative angle of arrival residual variance as a function of the knee frequency. The relative residual variance is the ratio of that residual variance which characterized the servo system precision to the variance of the incoming angle of arrival. Figure 9 shows that the performance of the servo system is highly sensitive to the turbulence conditions, represented here by the knee frequency. With a $10 \mathrm{~Hz}$ knee frequency and a $1 \operatorname{arcsec}^{2}$ angle of arrival variance, the theoretical pointing error (rms) thus equals 0.29 arcsec. Other simulations show that the accuracy is not really affected by the Signal-to-Noise Ratio, but rather by the bandwidth of the servo system, limited by the $6 \mathrm{~ms}$ command period.

\section{Results}

Analysis of the performance of the servo system has been made over eight months through October 1995. The results reported here concern "in situ" power spectra of an-

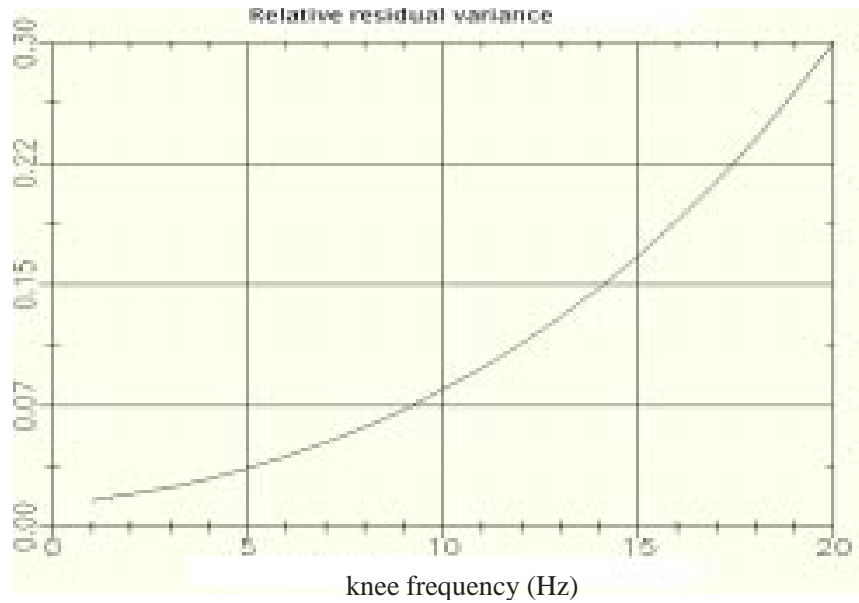

Fig. 9. Simulated variation of relative angle of arrival residual variance versus knee frequency

gle of arrival fluctuations, estimates of image tracking accuracy, and seeing parameters.

\subsection{Power spectra of the angle of arrival fluctuations}

In this section, we compare spectra predicted by Kolmogorov theory with experimental spectra. Influence of aperture size on the position of the knee frequency is also analyzed.

\subsubsection{Comparison of the Kolmogorov model with the experimental spectra}

The available pieces of information are the position signal of the mirror $y(t)$ and the residual error $\varepsilon(t)$. The transfer function in open loop, or gain, defined as the ratio of their spectral densities $W_{y}(f) / W_{\varepsilon}(f)$, leads to the bandwidth at $0 \mathrm{~dB} f_{0 \mathrm{~dB}}$. At this frequency, the residual error equals the correction signal (Demerlé et al. 1994), so that, beyond this frequency the correction process is no longer effective. Figure 10 shows an example of experimental spectral densities $W_{y}(f)$ and $W_{\varepsilon}(f)$, and the gain $W_{y}(f) / W_{\varepsilon}(f)$. This transfer function is relative to the movements about the horizontal axis of the Southern mirror during tracking of the star $\alpha$ Cygni (50 $10^{3}$ photons/sec), on October 7,1995 . $W_{y}(f)$ follows a $f^{-2 / 3}$ power law at low frequency and a $f^{-8 / 3}$ power law at high frequency. The knee frequency is approximately $6 \mathrm{~Hz}$, yielding a wind speed of nearly $5 \mathrm{~m} / \mathrm{s}$ from the empirical formula (Eq. (10)). $W_{y}(f) / W_{\varepsilon}(f)$ is fitted by the expected $1 / f^{2}$ curve. The frequency corresponding to $0 \mathrm{~dB}$ gain is approximately $15 \mathrm{~Hz}$.

From the bulk of recorded data, we have selected 91 temporal spectra of angle of arrival fluctuations. Selection was made on the basis of observation conditions considered as optimal (adjusted gains, cooled detectors).

Our observed spectra do not completely confirm the 

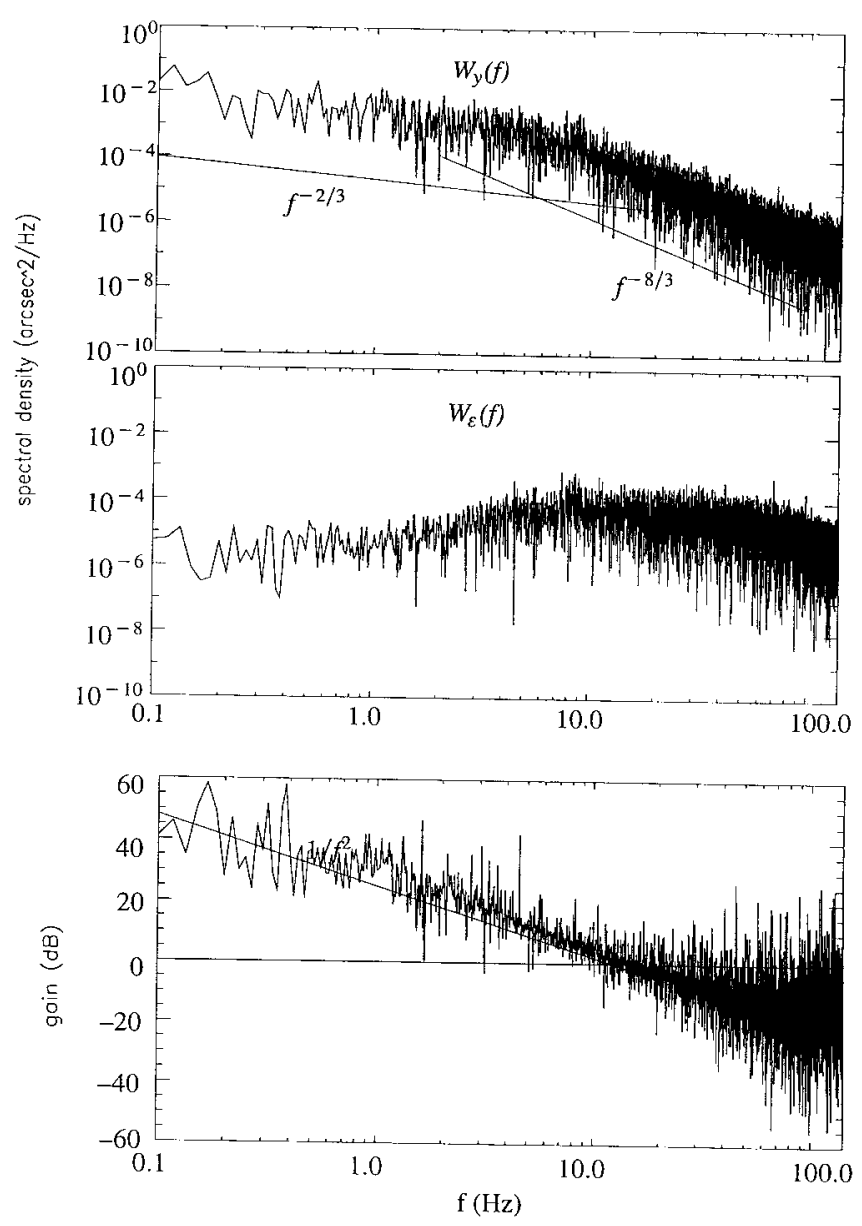

Fig. 10. Example of experimental spectral densities $W_{y}(f)$ and $W_{\varepsilon}(f)$, and transfer function $\left.W_{y}(f) / W_{\varepsilon} f\right)$

Kolmogorov prediction, but agreement with theory is quite satisfactory: all the spectra are in agreement with the $f^{-2 / 3}$ power law in the low frequency domain, while $67 \%$ of the spectra exhibit a $f^{-8 / 3}$ or $f^{-9 / 3}$ power law in the high frequency domain. The high frequency domain is influenced by noise causing it to rise above the expected $f^{-11 / 3}$ law. Moreover, on the mirror position signal the resonance of the closed loop transfer function also contributes to the presence of the $f^{-8 / 3}$ law. Up till now, in view of our results, we tend to consider that Kolmogorov predictions are relevant. Let us note that on the Sidney University Stellar Interferometer (SUSI), the same $f^{-8 / 3}$ behavior has been observed (ten Brummelaar \& Tango 1994).

\subsubsection{Knee frequency versus aperture size}

Since the diameter of the telescope entrance pupil can be reduced by using a mask, we can check the dependency of the knee frequency on the aperture size. To that aim, we analyzed files obtained on the same nights, with a $12 \mathrm{~m}$ baseline and two different pupil diameters, $26 \mathrm{~cm}$ (full pupil), and $11.5 \mathrm{~cm}$. The Northern mirror axis considered is the $X$-axis. Table 1 reports the aperture diameter $D$, the power laws followed by the considered spectra in both low frequency (LF) and high frequency (HF) domains, the knee frequency $f_{\text {knee, }}$, and the wind velocity $\mathcal{V}$ derived from Eq. (10).

Table 1. Three nights of observation on $\alpha$ Lyrae showing influence of pupil size on knee frequency $\left(r_{0}\right.$ between $9 \mathrm{~cm}$ and $10 \mathrm{~cm}$ at $600 \mathrm{~nm})$

\begin{tabular}{|c|c|c|c|c|c|c|}
\hline \multirow[t]{2}{*}{ Date } & \multirow{2}{*}{$\begin{array}{c}\text { UT } \\
\text { h:min }\end{array}$} & \multirow{2}{*}{$\begin{array}{c}D \\
\mathrm{~cm}\end{array}$} & \multicolumn{2}{|c|}{ power laws } & \multirow{2}{*}{$\begin{array}{c}f_{\text {knee }} \\
\mathrm{Hz}\end{array}$} & \multirow{2}{*}{$\begin{array}{c}\mathcal{V} \\
\mathrm{m} / \mathrm{s}\end{array}$} \\
\hline & & & LF & $\mathrm{HF}$ & & \\
\hline \multirow[t]{3}{*}{$07 / 21 / 95$} & $21: 20$ & 26 & $-2 / 3$ & $-8 / 3$ & 7 & 6.0 \\
\hline & $22: 13$ & 11.5 & $-2 / 3$ & $-8 / 3$ & 13 & 5.0 \\
\hline & $23: 47$ & 11.5 & $-2 / 3$ & $-8 / 3$ & 12 & 4.6 \\
\hline $07 / 22 / 95$ & 00:07 & 26 & $-2 / 3$ & $-8 / 3$ & 6 & 5.2 \\
\hline $06 / 25 / 95$ & $23: 37$ & 26 & $-2 / 3$ & $-10 / 3$ & 10 & 8.7 \\
\hline \multirow[t]{2}{*}{$06 / 26 / 95$} & $00: 30$ & 11.5 & $-2 / 3$ & $-11 / 3$ & 17 & 6.2 \\
\hline & $00: 52$ & 11.5 & $-2 / 3$ & $-11 / 3$ & 15 & 5.8 \\
\hline \multirow[t]{3}{*}{$06 / 08 / 95$} & 01:05 & 26 & $-2 / 3$ & $-8 / 3$ & 5 & 4.3 \\
\hline & 01:07 & 26 & $-2 / 3$ & $-9 / 3$ & 6 & 5.2 \\
\hline & 01:41 & 11.5 & $-2 / 3$ & $-10 / 3$ & 12 & 4.6 \\
\hline
\end{tabular}

Knee frequency is observed to grow by the expected factor of roughly 2 , when going from full aperture $(26 \mathrm{~cm})$ to masked aperture $(11.5 \mathrm{~cm})$. Since factor $f_{\text {knee }} D$ is quite stable through some given nights, as is the wind speed derived from Eq. (10), we can consider that the expected dependency is verified.

\subsubsection{Spectra obtained with internal source}

To verify that the measurements made on the sky are not affected by a spurious phenomenon originating on the table itself, we have recorded sequences of mirror position when an internal source on the table is observed.

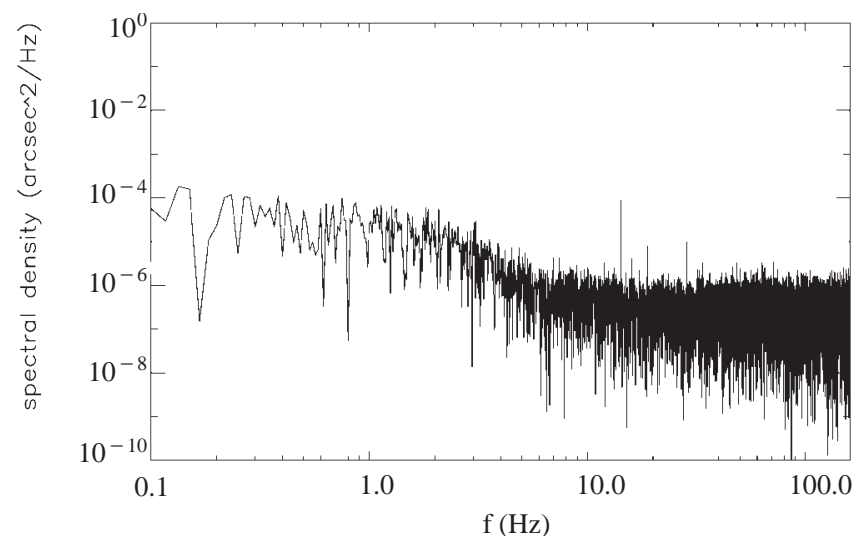

Fig. 11. Internal spectrum ( $X$-axis of the Southern mirror) 
Figure 11 shows a spectrum of internal fluctuations obtained on the horizontal axis of the Southern mirror. Exhibited spectrum is not completely flat and reveals low frequency contributions. The level of energy is 100 to 1000 times smaller than the one occurring in the spectrum of Fig. 10 when tracking a star. Therefore, we can assume that no additional perturbations occur on the table itself. However, since starlight has to travel in telescopes and delay lines (which is not the case for the light from internal source) we cannot conclude that the data recorded on a star are free from instrumental tip-tilt instabilities.

To interpret the low frequency contribution in the laboratory spectrum, we may invoke local instabilities in optical index of the air (temperature gradient, residual air flows), residual vibrations, or internally induced drift in beam orientation (an effect noticed a number of times in the course of adjusted procedures).

\subsection{Image tracking accuracy of the system}

The image tracking accuracy is sensitive to optimization of the bandwidth, to the turbulence conditions and to the signal to noise ratio.

\subsubsection{Tracking accuracy as a function of $r_{0}$}

Measurements of the servo-system performance have been made to evaluate influence of $r_{0}$ on the tracking accuracy using $\alpha$ Cygni, and baseline from $13 \mathrm{~m}$ to $17 \mathrm{~m}$. The flux conditions during the various observations were similar (flux ranging from $12410^{3}$ to $15010^{3}$ photons/sec Northward, and from $4210^{3}$ to $5110^{3}$ photons/sec Southward), but these measurements were carried out for different $r_{0}$ values. The latter was measured in open loop by ASSI and is relative to the star direction. Southward, the flux is lower than Northward, essentially due to the throughput of the external delay line. In addition, the Northern telescope is partly sheltered by the I2T building when working with small baselines. This implies that the signal to noise ratio, the turbulence conditions, and thus the servo system performance is usually better in the Northern channel. The tracking gains were optimized, and the PMTs cooled to a temperature close to $-5{ }^{\circ} \mathrm{C}$. Observations were carried out at full aperture $(D=26 \mathrm{~cm})$.

Tables 2 and 3 report the $0 \mathrm{~dB}$ gain frequency $f_{0} \mathrm{~dB}$ (open loop transfer function), the knee frequency $f_{\text {knee }}$ (Eq. (10)), the wind velocity $\mathcal{V}$ using Eq. (10), open loop Fried parameter $r_{0}$ OL, closed loop Fried parameter $r_{0} \mathrm{CL}$, and tracking error $(\mathrm{rms}) \sigma_{\varepsilon}$ on each axis. Some $f_{\text {knee }}$ values are not reported in the Tables since the following selection for this parameter is considered: disregarded are those for which part of the recording was taken during the motion of one telescope axis to keep the active mirror well within its dynamic range. Care must also be taken when $f_{\text {knee }}>f_{0} \mathrm{~dB}$. The measurements of $r_{0}$ CL are made from at least 10-second data sequences.

Some discrepancy exists between $r_{0} \mathrm{CL}$ and $r_{0} \mathrm{OL}$. Southward and Northward alike, $r_{0}$ CL is always larger than $r_{0}$ OL in these files. This result is partly due to the residual image motion being disregarded during image tracking. Northward, the discrepancy is lower than Southward where $r_{0}$ OL is usually more affected by a spurious drift of the Southern telescope. This effect is noticed on the image monitor. During the observations, we noticed a better reproducibility of $r_{0}$ oL Northward, and this parameter is therefore taken as being the most representative in the seeing process. However, we have to keep in mind that it also includes instrumental drifts and vibrations present in the Northern path.

Usually, tracking accuracy is more often better Northward than that Southward (lower than or equal to 0.2 arcsec). We can correlate this result with the measurement of the Fried parameter, which is also better in the Northern channel than in the Southern one.

We also notice that $f_{0} \mathrm{~dB}$ has to be larger than $10 \mathrm{~Hz}$ if optimal accuracy has to be reached. For example, October 8, at 18:45, Southward accuracy on the $Y$-axis reaches 0.11 arcsec at nearly $12 \mathrm{~Hz} f_{0 \mathrm{~dB}}$, as against 0.34 arcsec on the $X$-axis with a $5 \mathrm{~Hz} f_{0} \mathrm{~dB}$.

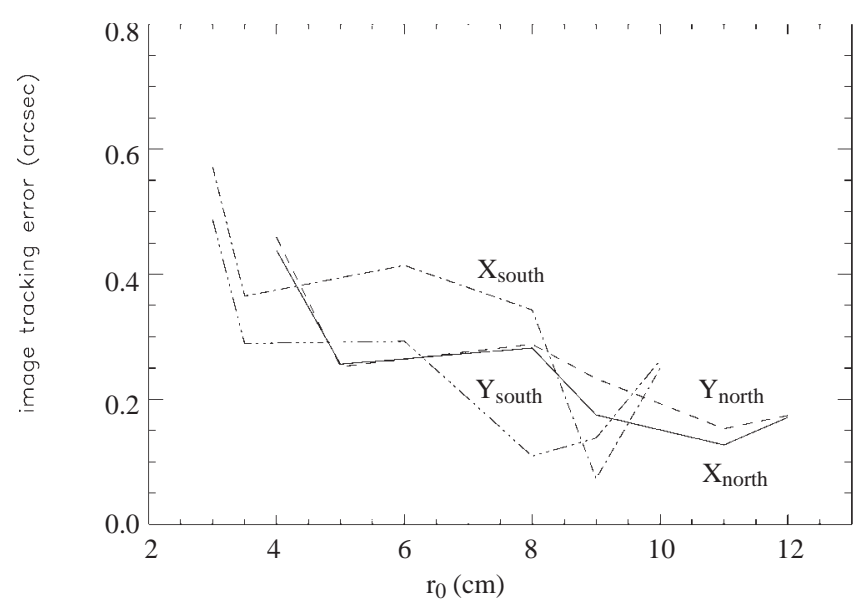

Fig. 12. Image tracking error versus open loop Fried parameter $r_{0}($ at $600 \mathrm{~nm})$

Figure 12 illustrates the observed tracking error as a function of Fried parameter $r_{0}$ OL for each mirror and each axis. For both motions, we note a significant degradation as $r_{0}$ ol becomes smaller than $8 \mathrm{~cm}$. Best accuracies reached on North mirror for the $X$ and $Y$ axes respectively are 0.13 arcsec and 0.15 arcsec. Similar figures for South mirror are 0.07 arcsec and 0.11 arcsec. Nevertheless, while this performance is currently met Northward, accuracies larger than 0.2 arcsec are more frequently estimated Southward.

From Fig. 12, the decrease of the error with the increase 
Table 2. Image tracking accuracy of the Northern mirror as a function of $r_{0}($ at $600 \mathrm{~nm})$ on the star $\alpha$ Cygni

\begin{tabular}{cc|rr|rr|rr|r|r|rc}
\hline date & $\mathrm{UT}$ & \multicolumn{2}{|c|}{$f_{0} \mathrm{~dB}(\mathrm{~Hz})$} & \multicolumn{2}{|c|}{$f_{\text {knee }}(\mathrm{Hz})$} & \multicolumn{2}{|c|}{$\mathcal{V}(\mathrm{m} / \mathrm{s})$} & $r_{0}(\mathrm{OL})$ & $r_{0}(\mathrm{CL})$ & \multicolumn{2}{|c}{$\sigma_{\varepsilon}(\operatorname{arcsec})$} \\
& h:min & \multicolumn{1}{|c|}{$X$} & $Y$ & \multicolumn{1}{|c|}{$X$} & $Y$ & \multicolumn{1}{|c|}{$X$} & $Y$ & $\mathrm{~cm}$ & $\mathrm{~cm}$ & \multicolumn{1}{c|}{$X$} & $Y$ \\
\hline $09 / 28 / 95$ & $18: 28$ & 17 & 15 & 15 & 15 & 13 & 13 & 4.0 & 3.8 & 0.438 & 0.459 \\
$09 / 27 / 95$ & $20: 25$ & 10 & 10 & 12 & - & 10 & - & 5.0 & 4.8 & 0.256 & 0.252 \\
$10 / 08 / 95$ & $19: 43$ & 20 & 25 & 20 & - & 17 & - & 8.0 & 11.8 & 0.282 & 0.288 \\
$10 / 08 / 95$ & $18: 45$ & 8 & 7 & 6 & 6 & 5 & 5 & 9.0 & 11.5 & 0.175 & 0.233 \\
$10 / 07 / 95$ & $18: 41$ & 12 & 10 & 6 & 6 & 5 & 5 & 11.0 & 18.4 & 0.127 & 0.153 \\
$09 / 21 / 95$ & $20: 17$ & 10 & 10 & 11 & 11 & 9.5 & 9.5 & 12.0 & 16.4 & 0.171 & 0.174 \\
\hline
\end{tabular}

Table 3. Image tracking accuracy of the Southern mirror as a function of $r_{0}$ (at $600 \mathrm{~nm}$ ) on the star $\alpha$ Cygni

\begin{tabular}{|c|c|c|c|c|c|c|c|c|c|c|c|}
\hline \multirow[t]{2}{*}{ date } & \multirow{2}{*}{$\begin{array}{c}\text { UT } \\
\text { h:min }\end{array}$} & \multicolumn{2}{|c|}{$f_{0 \mathrm{~dB}}(\mathrm{~Hz})$} & \multicolumn{2}{|c|}{$f_{\text {knee }}(\mathrm{Hz})$} & \multicolumn{2}{|c|}{$\mathcal{V}(\mathrm{m} / \mathrm{s})$} & \multirow{2}{*}{$\begin{array}{r}r_{0}(\mathrm{OL}) \\
\mathrm{cm}\end{array}$} & \multirow{2}{*}{$\begin{array}{r}r_{0}(\mathrm{CL}) \\
\mathrm{cm}\end{array}$} & \multicolumn{2}{|c|}{$\sigma_{\varepsilon}(\operatorname{arcsec})$} \\
\hline & & $X$ & $Y$ & $X$ & $Y$ & $X$ & $Y$ & & & $X$ & $Y$ \\
\hline$\overline{09 / 28 / 95}$ & $18: 28$ & $\overline{10}$ & 12 & 12 & 15 & $\overline{10}$ & 13 & 3.0 & 3.6 & 0.572 & 0.487 \\
\hline $09 / 27 / 95$ & $20: 25$ & 8 & 10 & 6 & 6 & 5 & 5 & 3.5 & 6.1 & 0.365 & 0.289 \\
\hline $10 / 08 / 95$ & $19: 43$ & 8 & 25 & 10 & 20 & 9 & 17 & 6.0 & 11.5 & 0.414 & 0.293 \\
\hline $10 / 08 / 95$ & $18: 45$ & 5 & 12 & 5 & 6 & 4 & 5 & 8.0 & 14.2 & 0.343 & 0.109 \\
\hline $10 / 07 / 95$ & $18: 41$ & 15 & 6 & - & 6 & - & 5 & 9.0 & 20.2 & 0.073 & 0.138 \\
\hline $09 / 21 / 95$ & $20: 17$ & 8 & 10 & - & 10 & - & 9 & 10.0 & 16.6 & 0.249 & 0.262 \\
\hline
\end{tabular}

of $r_{0}$ OL is quite apparent but there is not a similar rule with wind velocity (see Tables 2 and 3). Nevertheless, as accuracies obtained with a $12 \mathrm{~cm} r_{0}$ ol Northward and $8 \mathrm{~cm}$ Southward are not as good as those obtained with lower $r_{0}$ OL, this can be ascribed to wind velocity $(9 \mathrm{~m} / \mathrm{s}$ to compare with $5 \mathrm{~m} / \mathrm{s}$ ).

\subsubsection{Tracking accuracy as a function of flux}

In a search for a thread effect on fluxes, several stars with magnitude up to 2.2 have been observed. Table 4 gives the visible magnitude $m_{v}$ and the spectral type of these stars. Tracking errors of both mirrors versus flux are shown in Tables 5 and 6, and are plotted in one diagram (Fig. 13) (open loop $r_{0}$ estimates larger than $10 \mathrm{~cm}$ Northward and $8 \mathrm{~cm}$ Southward). Also presented is the frequency $f_{0 \mathrm{~dB}}$. For the Northern mirror, accuracy is roughly constant down to a flux of $3010^{3}$ photons/s. Southward accuracy is lower than that Northward for a flux of $4010^{3}$ photons/s and degradation is quite rapid. Even though the flux range in common for North and South is limited, it is apparent that accuracy does not behave similarly on the two channels. Moreover, the nearly permanent deficit in image quality undergone Southward increases the noise in the quad cell measurements (Eqs. (6) and (7)).

As discussed in Sect. 4.3, for 1 arcsec seeing, the theoretical tracking error after partial compensation for turbulence is on the order of 0.3 arcsec and not very sensitive to the noise level. This explains the behavior versus flux observed in Table 5 for the Northern mirror. For the Southern mirror, both the Signal-to-Noise Ratio and the bandwidth are lower than for the Northern mirror and this

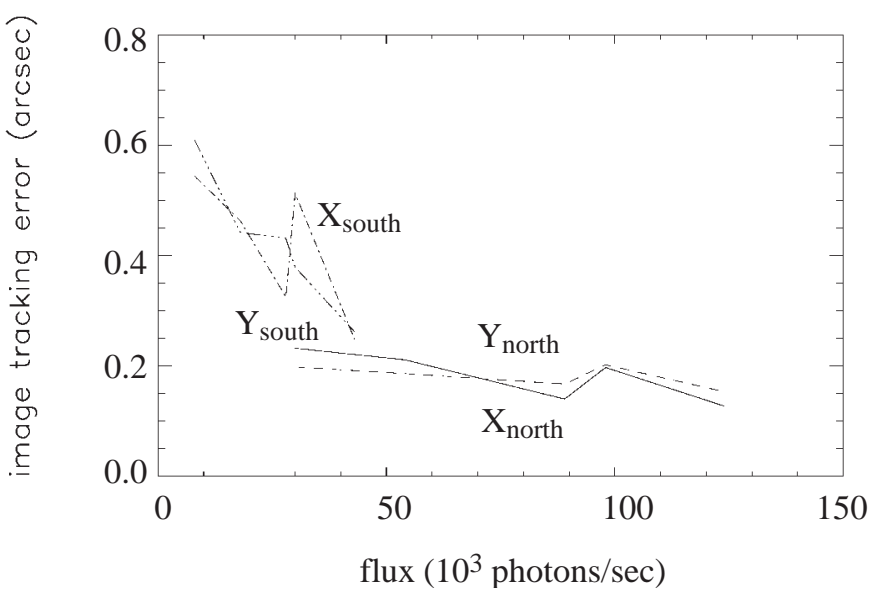

Fig. 13. Tracking error of Southern and Northern mirrors versus incident flux ( $r_{0}$ in Tables 5 and 6$)$

Table 4. List of observed stars

\begin{tabular}{lcc}
\hline star & $m_{v}$ & $\begin{array}{c}\text { spectral } \\
\text { type }\end{array}$ \\
\hline$\gamma$ Cyg & 2.2 & F8 \\
$\gamma$ And & 2.1 & K0 \\
$\beta$ And & 2.0 & M0 \\
$\alpha$ Per & 1.8 & F5 \\
$\alpha$ Cyg & 1.3 & A2
\end{tabular}


Table 5. Image tracking accuracy of the Northern mirror versus flux, $r_{0}$ estimated at $600 \mathrm{~nm}$

\begin{tabular}{lccc|rr|ll}
\hline Date & UT & flux & $r_{0}(\mathrm{OL})$ & $f_{0} \mathrm{~dB}$ & $(\mathrm{~Hz})$ & $\sigma_{\varepsilon}$ & (arcsec) \\
& h:min & $10^{3}$ photons $/ \mathrm{s}$ & $\mathrm{cm}$ & $X$ & $Y$ & $X$ & $Y$ \\
\hline $09 / 26$ & $19: 32$ & $30 \pm 4$ & 10 & 10 & 15 & 0.232 & 0.198 \\
$09 / 27$ & $01: 05$ & $54 \pm 5$ & 12 & 7 & 10 & 0.211 & 0.186 \\
$10 / 20$ & $22: 13$ & $89 \pm 9$ & 14 & 8 & 10 & 0.140 & 0.167 \\
$10 / 21$ & $01: 36$ & $98 \pm 11$ & 11 & 8 & 10 & 0.197 & 0.202 \\
$10 / 07$ & $18: 41$ & $124 \pm 12$ & 11 & 10 & 10 & 0.127 & 0.153 \\
\hline
\end{tabular}

Table 6. Image tracking accuracy of the Southern mirror versus flux, $r_{0}$ estimated at $600 \mathrm{~nm}$

\begin{tabular}{lccc|rr|ll}
\hline Date & UT & flux & $r_{0}(\mathrm{OL})$ & $f_{0} \mathrm{~dB}$ & $(\mathrm{~Hz})$ & $\sigma_{\varepsilon}$ & (arcsec) \\
& h:min & $10^{3}$ photons/s & $\mathrm{cm}$ & $X$ & $Y$ & $X$ & $Y$ \\
\hline $09 / 26$ & $19: 32$ & $8 \pm 2$ & 8 & 10 & 10 & 0.544 & 0.609 \\
$09 / 27$ & $01: 05$ & $18 \pm 2$ & 8 & 7 & 10 & 0.463 & 0.442 \\
$10 / 20$ & $22: 13$ & $28 \pm 5$ & 8 & 7 & 7 & 0.325 & 0.431 \\
$10 / 21$ & $01: 36$ & $30 \pm 5$ & 8 & 7 & 10 & 0.513 & 0.379 \\
$09 / 21$ & $20: 17$ & $43 \pm 5$ & 10 & 8 & 10 & 0.249 & 0.262 \\
\hline
\end{tabular}

could justify the worse performance as shown in Table 6 . Moreover, the contribution of the noise as deduced from Eqs. (6) and (7) is not sufficient to explain the difference in performance. More observations are necessary if progress is to be made on this subject.

In summary, we can say that a typical accuracy of 0.2 arcsec is achievable when $r_{0}$ is larger than $8 \mathrm{~cm}$, and the flux greater than $3010^{3}$ photons/s.

\subsection{Seeing measurements}

A set of observations recorded on 11 nights distributed between July and October 1995, and another set of 8 observations distributed between February and July 1995, enable statistic analysis of parameters $r_{0}$ and $t_{0}$.

\subsubsection{Fried parameter}

For the first set, we have selected observations for which estimates of $r_{0}$ in open loop mode and in closed loop mode are available both Northward and Southward. The open loop mode is considered as reliable as soon as it yields reproducible estimates. Besides, in order to somewhat enrich the statistic, we used the second set of observations for which only estimated $r_{0}$ values obtained in closed loop mode are available. Figure 14 shows occurrences of open loop (solid lines) and closed loop (dashed lines) $r_{0}$ Northward and Southward.

Northward, both histograms show a predominance appearing for $r_{0}$ ranging from $10 \mathrm{~cm}$ to $12 \mathrm{~cm}$. The open loop estimates do not exceed $14 \mathrm{~cm}$, although the closed loop ones rise up to $20 \mathrm{~cm}$ and present one value at $25 \mathrm{~cm}$. Southward, the open loop estimates are continuously distributed from $2 \mathrm{~cm}$ to $12 \mathrm{~cm}$. They are generally lower
Southward than Northward, probably due to the spurious drift of the Southern telescope, usually stronger than the Northern one. The closed loop estimates show a predominance around $10 \mathrm{~cm}$. Same as Northward, the latter can reach values up to $20 \mathrm{~cm}$, unlike the open loop ones which never do.

As we already mentioned, we could suspect the system to over-estimate the closed loop $r_{0}$ as the image tracking is not perfect and residual error remains. But let us consider the case for which closed loop $r_{0}$ reaches $25 \mathrm{~cm}$ Northward and $30 \mathrm{~cm}$ Southward. The respective open loop $r_{0}$ are $11 \mathrm{~cm}$ and $9 \mathrm{~cm}$. The values of respective parameters are as follows: tracking accuracy at 0.19 arcsec and 0.15 arcsec (included $X$ and $Y$ axes) and $0 \mathrm{~dB}$-frequency at $12 \mathrm{~Hz}$ and $15 \mathrm{~Hz}$. Since these parameters are favorable for the relevance of closed loop $r_{0}$ estimates, we can thus expect the latter to be realistic. So, in this case, the tracking imperfection does not seem to be the main cause of the discrepancy between closed loop and open loop $r_{0}$. In fact, over-estimation of closed loop $r_{0}$ due to imperfect tracking and under-estimation of open loop $r_{0}$ due to telescope drifts should jointly contribute to these discrepancies. In order to know which one is the best estimate, it would be very interesting to perform a cross calibration with measurements made by the Nice University team, currently in charge of the Grating Scale Monitor (GSM) experiment on one of the rails of $\mathrm{I} 2 \mathrm{~T}$. This experiment is intended to measure temporal and spatial characteristics of the wavefront (Ziad 1993; Agabi 1994). Their observations, carried out on few short periods (of a few nights each) distributed over the year, were not simultaneous to ours. An alternative way to estimate $r_{0}$ could be to sum the mirror position and the quad-cell error, but with a required proper 

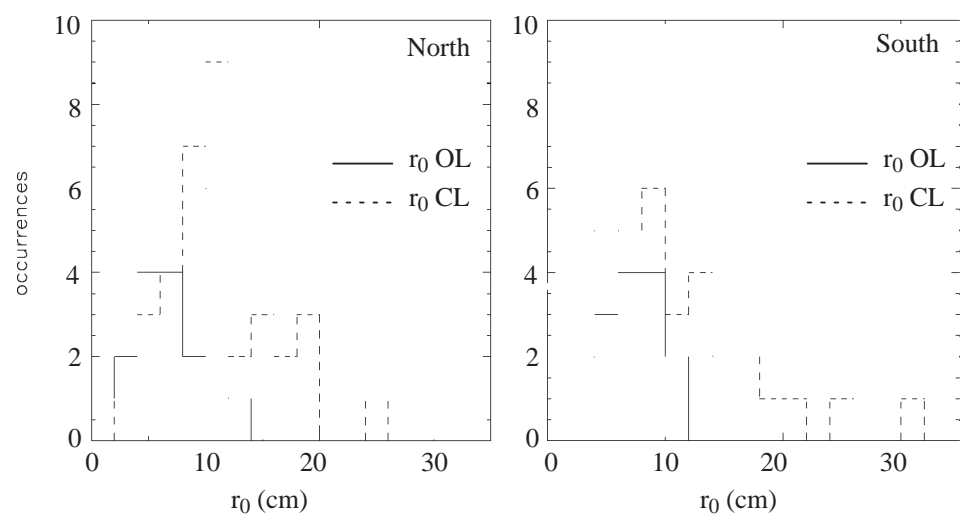

Fig. 14. Occurrences of open loop (solid lines) and closed loop (dashed lines) estimates of Northward and Southward $r_{0}$ (at $600 \mathrm{~nm})$
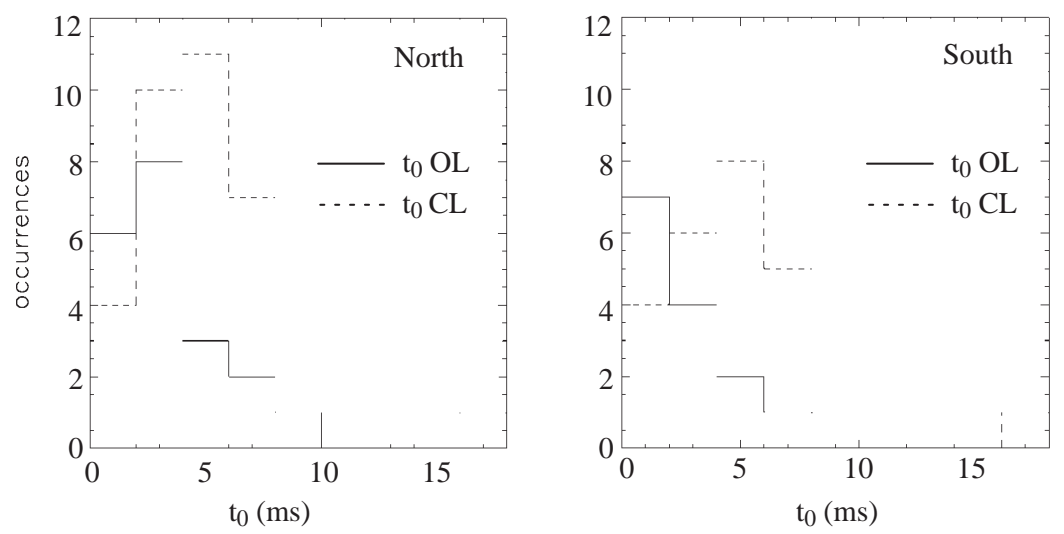

Fig. 15. Occurrences of open loop (solid lines) and closed loop (dashed lines) estimates of Northward and Southward $t_{0}$ (at $600 \mathrm{~nm})$

calibration of the photon noise. This could be envisaged for future work.

\subsubsection{Coherence time}

From $r_{0}$ and wind speed estimates, we can deduce coherence time $t_{0}$ (Eq. (11)). We have built histograms as shown in Fig. 15, for which the whole set of observations was used. Coherence times obtained from open loop $r_{0}$ and from closed loop $r_{0}$ have been distinguished. Northward, we see that $t_{0}$ stands near $3 \mathrm{~ms}$ most of the time in the open loop case and near $5 \mathrm{~ms}$ in the closed loop case. Southward, the behavior of $t_{0}$ in the closed loop case is similar to that Northward but the predominance in the open loop case is only $1 \mathrm{~ms}$. The open loop case is more pessimistic than the closed loop one due to the low $r_{0}$ values. Though these values should be reviewed after calibration of $r_{0}$, they tend to confirm that our records of dispersed fringes (Robbe et al. 1994), using a $20 \mathrm{~ms}$ exposure time, are not exactly entered into the short-exposure mode. Nevertheless, the histogram shows that $t_{0}$ longer than $10 \mathrm{~ms}$ sometimes happen in the closed loop case Northward as Southward.

These values can be compared with those measured by other instruments. Nightingale \& Buscher (1991) give median values of $5 \mathrm{~ms}$ and $10 \mathrm{~ms}$ at $500 \mathrm{~nm}$, obtained during two different nights at La Palma Observatory in the Canary Islands. These values correspond to $6.2 \mathrm{~ms}$ and $12.4 \mathrm{~ms}$ at $600 \mathrm{~nm}$. A mean value of $5 \mathrm{~ms}$ at $492 \mathrm{~nm}$ is given by Davis et al. (1995) at the Paul Wild Observatory, Australia, as equivalent to $6.3 \mathrm{~ms}$ at $600 \mathrm{~nm}$. On the same site, Davis \& Tango (1995) report values from less than $1 \mathrm{~ms}$ to $7 \mathrm{~ms}$ at $443 \mathrm{~nm}$, equivalent to less than $1.4 \mathrm{~ms}$ to $10 \mathrm{~ms}$ at $600 \mathrm{~nm}$. Colavita et al. (1987) at Mt Wilson, U.S.A., give $T_{0}$ values from $11 \mathrm{~ms}$ to $18 \mathrm{~ms}$ at $550 \mathrm{~nm}$, but as their definition is a factor 2.58 larger than ours (Eq. (11)) their measurements are equivalent to $t_{0}$ from $4.7 \mathrm{~ms}$ to $7.7 \mathrm{~ms}$ at $600 \mathrm{~nm}$. Our values are as good as or lower than the ones measured at other observatories. Anyhow, this modest statistic confirms the need for servo-control systems on the I2T interferometer, even though typical conditions of turbulence on the site are not quantified on a large temporal basis. 


\section{Conclusion}

In this paper, the ASSI recombining table has been presented. The star tracker is described in details. It is made of two tip-tilt mirrors (one per telescope) and one quad cell equipped with four PMTs. The servo-loop of this system is characterized by its transfer functions. The closed loop bandwidth at $-3 \mathrm{~dB}$ is $63 \mathrm{~Hz}$ while the rejection bandwidth at $0 \mathrm{~dB}$ is $22 \mathrm{~Hz}$. The theoretical behavior of the quad cell signals is presented, showing the requirement of a loop gain adjustment versus the seeing conditions. The theoretical behavior of the temporal power spectrum of the turbulence angle of arrival is also given. We emphasize the knee frequency of this spectrum which allows a rough estimation of the average wind speed in the atmosphere. While the power of the spectrum is directly related to $r_{0}$. We were able to estimate both $r_{0}$ and wind speed using the angle of arrival data recorded in closed loop operation, allowing a full characterization of the observation conditions.

Performance of the ASSI star tracker was measured on the sky during a few nights. It allowed to bring a significant gain in visibility measurements on the $\mathrm{I} 2 \mathrm{~T}$, which will be presented in a future paper. The wavefront angle of arrival correction system on the I2T + ASSI instrument proves to be able to reach good performance in terms of image tracking accuracy, $\pm 0.2 \operatorname{arcsec}\left(r_{0} \geq 10 \mathrm{~cm}\right.$ at $\left.600 \mathrm{~nm}\right)$. The residual wavefront angles of arrival cause a reduction in the fringe visibility measurements. The rms visibility resulting from losses caused by small angle of arrival errors when the beams are combined are given by Buscher (1988). The \pm 0.2 arcsec angle of arrival error implies that the two beams are kept to within one third the size of the Airy disc radius at $0.85 \mu \mathrm{m}$, and ensures visibility loss below $15 \%$.

We observed a permanent deficit in tracking accuracy for the South channel at relatively low light level which will require to be fixed in a near future.

Most of the measured temporal spectra of angle of arrival exhibit a behavior compatible with the Kolmogorov theory, in terms of power law.

Estimates of $10 \mathrm{~cm}$ for the Fried parameter $r_{0}$ and $5 \mathrm{~ms}$ for the coherence time $t_{0}$ at $600 \mathrm{~nm}$ have been met in our observations, and are presently considered as average values on the site, yet requiring a better statistic. Such values are comparable with those obtained on other sites (Colavita et al. 1987; Nightingale \& Buscher 1991; Davis et al. 1995).

Acknowledgements. This work has been supported by contracts from "Direction des Recherches, Etudes et Techniques (DRET)" of the Defense Minister, France. We wish to thank C. Coudrain and G. Merlin for their helpful interventions on the instrument.

\section{References}

Agabi A., 1994, PhD Thesis, University of Nice

Bonneau D., 1979, A\&A 80, 11

Buscher D.F., 1988, PhD Thesis, Cambridge University

Cassaing F., Mugnier L., Sorrente B., Coudrain C., Rousset G., 1995, ONERA Technical Report 4/4625

Colavita M.M., Shao M., Staelin D.H., 1987, Appl. Opt. 26, 4106

Conan J.-M., 1994, PhD Thesis, University of Paris XI Orsay

Conan J.-M., Rousset G., Madec P.-Y., 1995, J. Opt. Soc. Am. 7,1559

Coulman C.E., Vernin J., Coqueugniot Y., Caccia J.L., 1988, Appl. Opt. 30, 118

Damé L., Faucherre M., Bourdet G., et al., 1988, Proc. of ESA Workshop on Optical Interferometry in Space, SP-273, 11

Davis J., Lawson P.R., Booth A.J., Tango W.J., Thorvaldson E.D., 1995, MNRAS 273, L53

Davis J., Tango W.J., 1995 (preprint)

Demerlé M., Madec P.-Y., Rousset G., 1994, in Adaptive Optics for Astronomy, Alloin D.M. and Mariotti J.-M. (eds.) NATO ASI Ser. C 423, 73

Di Benetto G.P., Rabbia Y., 1987, A\&A 188, 114

Faucherre M., Bonneau D., Koechlin L., Vakili F., 1983, A\&A 120,263

Gerson G., Rue A.K., 1989, in the Infrared Handbook, Wolfe W.L. and Zissis G.J. (eds.). Environmental Research Institute of Michigan, Chapitre 2

Hogge C.B., Butts R.R., 1976, IEEE Trans. Antennas Propag., AP-24 2, 144

Koechlin L., 1985, PhD Thesis, University of Nice

Koechlin L., Rabbia Y., 1985, A\&A 153, 91

Koehler B., Manil E., 1990, Proc. of SPIE Symp. on Advanced Technology Optical Telescopes IV, 1236, 463

Labeyrie A., 1975, A\&A 162, 71

Ma J., Sun H., Wang J., Yan D., 1989, Appl. Opt. 28, 2123

Martin H.M., 1987, PASP 99, 1360

Nightingale N.S., Buscher D.F., 1991, MNRAS 251, 155

Robbe S., Sorrente B., Cassaing F., et al., 1994, Proc. of SPIE Symp. on Amplitude and Intensity Spatial Interferometry, 2200, 222

Roddier F., 1981, Progress in Optics, XIX, Wolf. E. (ed.). North Holland Publish, 281

Roddier F., Northcott M.J., Graves J.E., McKenna D.L., 1993, J. Opt. Soc. Am. 10, 957

Rousset G., 1994, in Adaptive Optics for Astronomy, Alloin D.M. and Mariotti J.M. (eds.), NATO ASI Ser. C 423, 115

Schumacher G., Cruzalèbes P., Jeanne A., Rabbia Y., 1991, Proc. of ESO Conf. on High-Resolution Imaging by Interferometry 39, 715

Shao M., Colavita M.M., Hines B.E., et al., 1988, A\&A 93, 357

Sorrente B., Cassaing F., Béal D., et al., 1991, Proc. of ESO Conf. on High-Resolution Imaging by Interferometry 39 , 1133

Tatarski V.I., 1971, Israel Program of Scientific Translation

ten Brummelaar T., Tango W.J., 1994, Experim. Astron. 4, 297

Tyler G.A., Fried D.L., 1982, J. Opt. Soc. Am. 72, 804

Yura H.T., 1973, J. Opt. Soc. Am. 63, 567

Ziad A., 1993, PhD Thesis, University of Nice 